ARTICLE

https://doi.org/10.1038/s41467-019-10586-2

\title{
The hydrolase Lpql primes mycobacterial peptidoglycan recycling
}

Patrick J. Moynihan (D) ${ }^{1}$, lan T. Cadby ${ }^{1}$, Natacha Veerapen', Monika Jankute', Marialuisa Crosatti Galina V. Mukamolova ${ }^{2}$, Andrew L. Lovering ${ }^{1} \&$ Gurdyal S. Besra (1) ${ }^{1}$

Growth and division by most bacteria requires remodelling and cleavage of their cell wall. A byproduct of this process is the generation of free peptidoglycan $(P G)$ fragments known as muropeptides, which are recycled in many model organisms. Bacteria and hosts can harness the unique nature of muropeptides as a signal for cell wall damage and infection, respectively. Despite this critical role for muropeptides, it has long been thought that pathogenic mycobacteria such as Mycobacterium tuberculosis do not recycle their PG. Herein we show that $M$. tuberculosis and Mycobacterium bovis BCG are able to recycle components of their PG. We demonstrate that the core mycobacterial gene Ipql, encodes an authentic NagZ $\beta$ - $N$-acetylglucosaminidase and that it is essential for PG-derived amino sugar recycling via an unusual pathway. Together these data provide a critical first step in understanding how mycobacteria recycle their peptidoglycan.

\footnotetext{
${ }^{1}$ Institute of Microbiology and Infection, School of Biological Sciences, University of Birmingham, Birmingham B15 2TT, UK. ${ }^{2}$ Leicester Tuberculosis Research group, Department of Respiratory Sciences, University of Leicester, Leicester LE1 9HN, UK. Correspondence and requests for materials should be addressed to P.J.M. (email: p.j.moynihan@bham.ac.uk)
} 
T he cell wall of Mycobacterium tuberculosis is built upon a foundation of peptidoglycan (PG). The remainder of this structure is formed by the modification of muramic acid residues with an arabinogalactan polymer that is in turn esterified by mycolic acids ${ }^{1}$. This waxy coating not only contributes to drug resistance in $M$. tuberculosis, but is also the target of several mycobacteria-specific antibiotics ${ }^{1}$. The challenge of multi- and extensively drug-resistant $M$. tuberculosis has not adequately been met by drug discovery efforts, however, recent reports suggest that $\beta$-lactams are effective in treating these drug-resistant infections $^{2-4}$. Despite their therapeutic promise, we know relatively little about the turnover of PG in mycobacteria, which is the eventual target of $\beta$-lactam antibiotics ${ }^{5}$.

For most bacteria, maintenance of a PG sacculus is an essential aspect of life. PG is a heteropolymer comprised of glycan chains with a repeating disaccharide motif of $\mathrm{N}$-acetylglucosamine $\beta 1 \rightarrow 4 N$-acetylmuramic acid (GlcNAc-MurNAc), which are then cross-linked to one another via short peptides attached to the $\mathrm{C}-3$ D-lactyl moiety of MurNAc (Fig. 1a). The integrity of this macromolecule must be maintained under most growth conditions and its rupture leads to lysis and cell death ${ }^{6}$. As a result of this essentiality, it is vital that cells are able to withstand their own internal turgor pressure and still be able to cleave the cell wall to allow for division, growth, and the insertion of macromolecular structures such as secretion systems ${ }^{6}$. Throughout this process, the activity of lytic enzymes or through the attack of host agents like lysozyme, the sacculus is cleaved with the resulting generation of small PG fragments ${ }^{7}$.

In Gram-positive bacteria, muropeptides are typically released from the cell wall through the action of lysozyme-like hydrolytic enzymes, whereas in Gram-negative bacteria, lytic transglycosylases generate 1,6-anhydroMurNAc products ${ }^{8,9}$. These metabolites have been shown to be important in many aspects of host-pathogen interactions. For example, tracheal cytotoxin produced by Bordetella pertussis is the product of lytic transglycosylases $^{10}$. Release of a similar molecule has also been shown to be involved in tissue damage during Neisseria gonorrhoeae infection and in the closure of the light-organ of the bobtail squid ${ }^{11,12}$. In many organisms, soluble PG acts as a potent immune stimulator once sensed by NOD receptors and other pattern recognition receptors ${ }^{13}$

Aside from host organisms, PG metabolites are also important signalling molecules for the bacteria themselves. Recycling of PG has been studied in great detail in a small number of organisms including Escherichia coli, Pseudomonas aeruginosa, and Bacillus subtilis among others ${ }^{14}$. The recycling pathway typically involves the step-wise degradation of the polymer into its monomeric constituents, monosaccharides, and amino acids (Fig. 1b). Despite common biochemical steps, compartmentalisation of these steps tends to be organism specific ${ }^{8}$. The resulting monosaccharides are eventually phosphorylated and MurNAc-6-phosphate is converted into glucosamine-6-phosphate through the activities of cytoplasmic MurQ and NagA enzymes (Fig. 1b). At the same time, the stem peptides are degraded to smaller components and typically shunted back into PG biogenesis. Growth on MurNAc as a sole carbon source has never been demonstrated for a bacterium that lacks MurQ. Furthermore, recycling of MurNAc in a bacterium that lacks MurQ has only been described in Pseudomonas putida, and many bacteria, including mycobacteria, are not thought to recycle their PG at all ${ }^{15}$

In the present study, we sought to determine whether mycobacteria are capable of recycling their PG and if so, what impact this has on the bacterium. We demonstrate that these bacteria do indeed possess the biochemical capacity to recycle PG elements and determined how the bacteria generate free MurNAc for recycling. Our data indicate that loss of a key recycling enzyme, LpqI, leads to increased antibiotic and lysozyme resistance. a

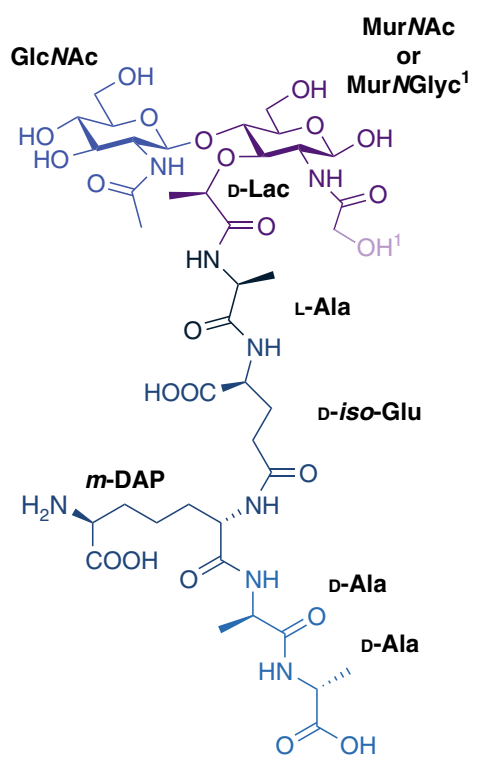

b

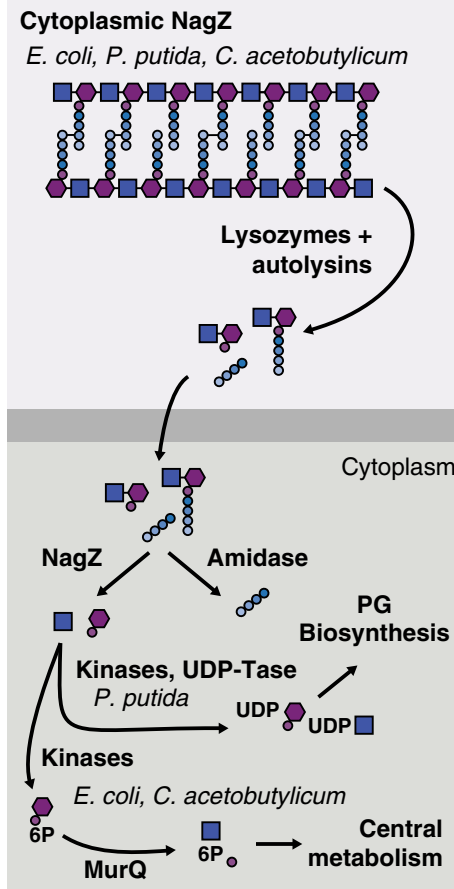

Extracellular NagZ B. subtilis

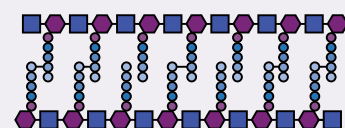

Lysozymes + autolysins

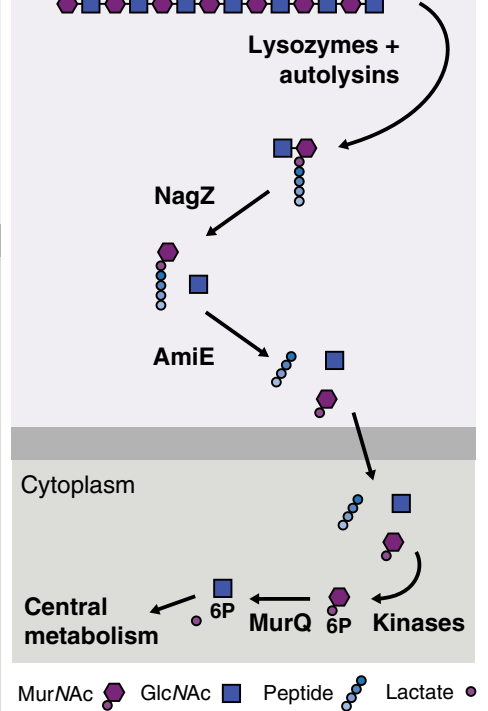

Fig. 1 Overview of PG recycling. a The basic building block of PG is GIcNAc-MurNAc-pentapeptide. Enzymes produced by the bacterium or the host are able to cleave every major linkage in PG. b Known PG-recycling machinery is variable with respect to the localisation of NagZ and the subsequent conversion to GIcNAc-1P or UDP-GIcNAc/MurNAc. All known MurNAc recovery systems that sustain bacterial growth (as opposed to strictly recycling e.g. P. putida) terminate at MurQ in the cytoplasm 


\section{Results}

PG-recycling genes in mycobacteria. The genome of $M$. tuberculosis encodes many lytic enzymes, including at least five resuscitation-promoting factors (Rpfs) and $>10$ peptidases and amidases in addition to penicillin-binding proteins with potential lytic activities ${ }^{16}$. The Rpfs are most likely lytic transglycosylases with the product of $\mathrm{RpfB}$ having been recently confirmed as a GlcNAc-1,6-anhydroMurNAc disaccharide-peptide ${ }^{17}$. Although $M$. tuberculosis does appear to encode at least one lysozyme, Rv2525, its activity has not been demonstrated ${ }^{18}$. A recent comparative study of PG-active enzymes in mycobacteria indicated that although significant differences exist, enzymes that can likely degrade all of the major covalent linkages of PG are encoded in the genomes of all mycobacteria ${ }^{16}$. The products of most of these enzymes have not been experimentally demonstrated, however, their conservation underscores the importance of PG-remodelling during growth and division of mycobacteria.

Most autolytic enzymes produce small PG metabolites (muropeptides), indicating that mycobacteria should generate these molecules during the course of normal growth. Indeed, soluble PG fragment release has been observed for both $M$. smegmatis and $M$. tuberculosis in vitro ${ }^{19,20}$. Given the slow release of PG fragments by mycobacteria, we evaluated the presence of known PG-recycling systems in the genome of several corynebacterial species (Supplementary Table 2) ${ }^{15,21}$. BLAST analysis of the Corynebacterium glutamicum, M. tuberculosis, Mycobacterium leprae, and M. bovis BCG genomes indicates that they lack genes related to any known muropeptide import proteins, PGmetabolite phosphorylation systems, and murQ. The only sugarkinase orthologs identified in the genome have previously been characterised as glucose-kinases although they have not been directly tested for amino sugar-phosphotransferase activity ${ }^{22}$. This contrasts sharply with $M$. smegmatis for which an apparently complete "classical" muropeptide recovery system exists, making it a poor model for the PG metabolism of $M$. tuberculosis (Supplementary Table 2). Taken together, the available data indicates that $M$. tuberculosis and almost all other mycobacteria lack most of the known PG-recycling genes from other bacteria, with only two conserved genes potentially associated with PGrecycling, (nagA-Rv3332, nagZ/lpqI-Rv0237).

Biochemical and structural characterisation of LpqI. In previously characterised PG-recycling systems free amino sugars are produced by NagZ, which belongs to the CAZy glycoside hydrolase family $3(\mathrm{GH} 3)^{23}$. This family is a large group of enzymes that have hydrolytic and phosphorylytic activity and remove $\beta$-linked sugars from proteins and polysaccharides ${ }^{24,25}$. The $\beta$ - $N$-acetylglucosaminidase sub-family including all known NagZ enzymes utilise a conserved Asp-His catalytic dyad, which has been well characterised 26,27 . A BLAST search of the $M$. tuberculosis H37Rv genome revealed only one NagZ ortholog, which was previously named LpqI in light of its identification as a lipoprotein including an appropriately positioned lipobox at the $\mathrm{N}$-terminus of the protein ${ }^{28}$. As a lipoprotein LpqI is expected to be found attached to the external face of the cytoplasmic membrane, which is consistent with proteomics results ${ }^{28}$. LpqI has also been identified as a likely mannosylated glycoprotein in a proteomics screen using ConA chromatography ${ }^{29}$. The lpqI gene is found in all mycobacteria with sequenced genomes including $M$. leprae, which has a substantially reduced genome suggesting that it is involved in a conserved process across all mycobacteria (Supplementary Table 2, Supplementary Fig. 1).

Given the absence of other PG-recycling-associated genes, we sought to identify the function of LpqI. Although LpqI bears significant sequence similarity to known $\beta$ - $N$-acetylglucosaminidases, recent studies have demonstrated that divergent activities for this sub-family of enzymes are possible ${ }^{25}$. These activities included the ability to release sugars other than GlcNAc from reporter substrates and apparent phosphorolytic activity. To test this, we cloned, expressed and purified $\mathrm{LpqI}_{\mathrm{TB}}$ using an $\mathrm{N}$-terminal $\mathrm{His}_{6}$-SUMO tag, which was subsequently cleaved from the protein. We first determined whether the protein was in fact a $\beta-N$-acetylglucosaminidase by testing its activity on a variety of substrates including many sugars that would be found in the cell wall of mycobacteria. Using convenient reporter sugars we assessed the ability for the enzyme to release $p$-nitrophenolate or 4 methylumbeliferone from a variety of conjugated sugars (Fig. 2a). Although not exhaustive, this set of sugars covers most major modifications to the cell wall including the AG itself, $O$-mannose modifications of proteins, GalN modification of arabinan, the rhamnose-linker sugar of AG and the GlcNAc and GlcN found in PG. The only detectable activity for LpqI was with GlcNAccontaining substrates (Fig. 2a). Critically, in mycobacteria, this sugar is limited to the backbone of PG and a small amount in the linker unit (MurNAc-6-P-Rha-GlcNAc-galactan) between PG and arabinogalactan. The Michaelis-Menten constants $\left(k_{\mathrm{cat}}=\right.$ $2.0 \times 10^{-2} \pm 0.04 \times 10^{-2} \mathrm{~s}^{-1}$ and $\left.K_{\mathrm{m}}=72 \pm 5 \mu \mathrm{M}\right)$ of LpqI using $4 \mathrm{MU}-\mathrm{GlcNAc}$ as a substrate were found to be similar to other NagZ enzymes using this substrate (Fig. 2b) ${ }^{26}$. In a similar assay we were also able to show that LpqI releases GlcNAc from soluble PG fragments (Fig. 2c), including PG fragments with a 1,6anhdroMurNAc terminus (Supplementary Fig. 2). Although hydrolytic activity has been reported for most NagZ-type enzymes, a recent report suggested that $\beta-N$-acetylglucosaminidases from the GH3 family are in fact phosphorylases ${ }^{25}$. Another GH3 $\beta-N$-acetylglucosaminidase was recently reported to lack this latter activity, suggesting that it may not be a general property of the family ${ }^{30}$. We tested the activity of the enzyme under the same conditions as reported previously $(50 \mathrm{mM}$ sodium phosphate buffer, $\mathrm{pH} 7.0,300 \mathrm{mM} \mathrm{NaCl}, 5 \mathrm{mM} \mathrm{MgSO} 4,10 \%$ glycerol, $1 \mathrm{mM}$ DTT) for Nag3 from Celulomonas fimi and found that there was no detectable difference with our observed hydrolytic activity. The product of the reaction also comigrated with GlcNAc on TLCs and not GlcNAc-1-P (Supplementary Fig. 3).

To confirm that LpqI is an exo-acting enzyme rather than an autolytic $\beta-N$-acetylglucosaminidase we evaluated the activity of the enzyme using defined-length chitooligosaccharides. As shown in Supplementary Fig. 4a, LpqI produces GlcNAc ${ }_{5}$ and GlcNAc from $\mathrm{GlcNAc}_{6}$. This is in contrast to the known endo-acting chitinase ChitB, which produces $\mathrm{GlcNAc}_{2}$ and $\mathrm{GlcNAc}_{4}$ from the same substrate ${ }^{31}$. We further tested the ability of the enzyme to act as an autolysin and release soluble PG from insoluble material by incubating the enzyme with fluorescently labelled PG. In this assay, LpqI was not observed to release any soluble PG in contrast to the muramidase mutanolysin (Supplementary Fig. 4b). Together these data support the role of LpqI as an exo-acting enzyme involved in fragment recycling, rather than as an endoacting autolysin.

To further confirm its function and validate its role in PGrecycling, we solved the $1.96 \AA \mathrm{X}$-ray crystal structure of LpqI (PDB code: 6GFV; Supplementary Fig. 1, Supplementary Table 3). LpqI consists of a single TIM-barrel domain similar to cytoplasmic Gram-negative orthologs but lacks the C-terminal domain associated with extracellular NagZ enzymes from some Gram-positive bacteria (Supplementary Fig. 5). Alignment of LpqI with the NagZ/GlcNAc/1,6-anhydroMurNAc complex from $P$. aeruginosa $\left(\mathrm{NagZ}_{\mathrm{Pa}}\right.$; $\mathrm{PDB}: 5 \mathrm{G} 3 \mathrm{R}$ ) or NagZ from $B$. subtilis (PDB:4GYJ) using the SSM Superpose function in COOT resulted in a root-mean-square deviation of $2.30 \AA$ and $1.63 \AA$, respectively (Supplementary Fig. 5). Superposition of the post-cleavage 

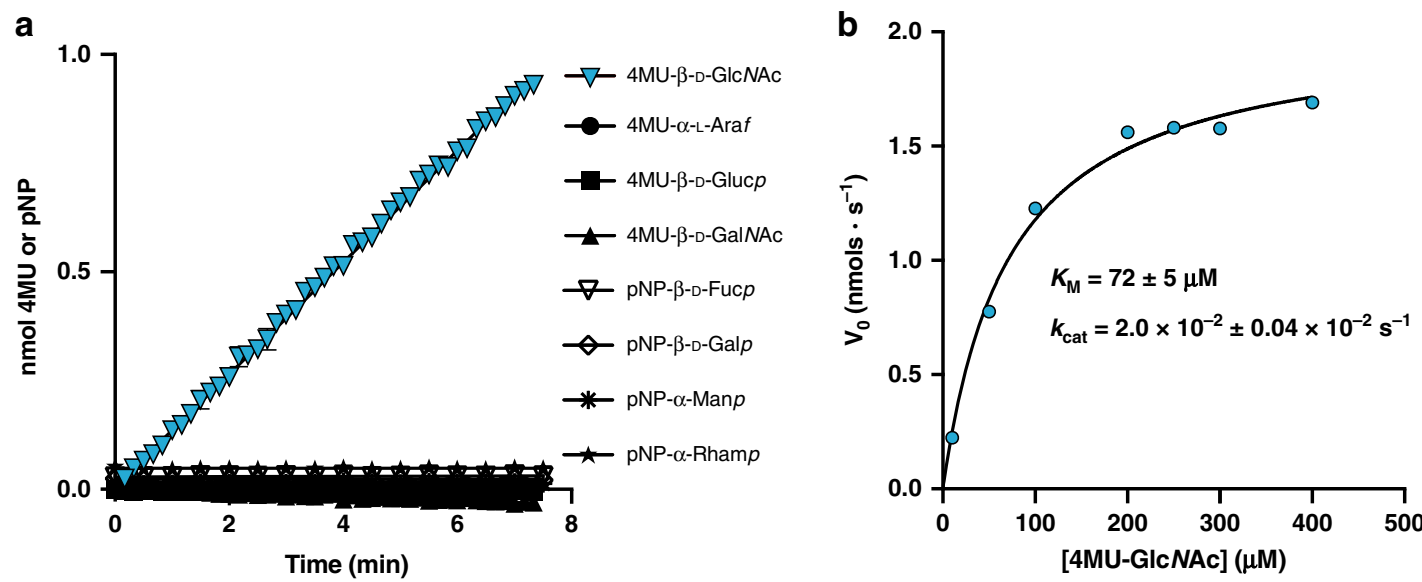

C
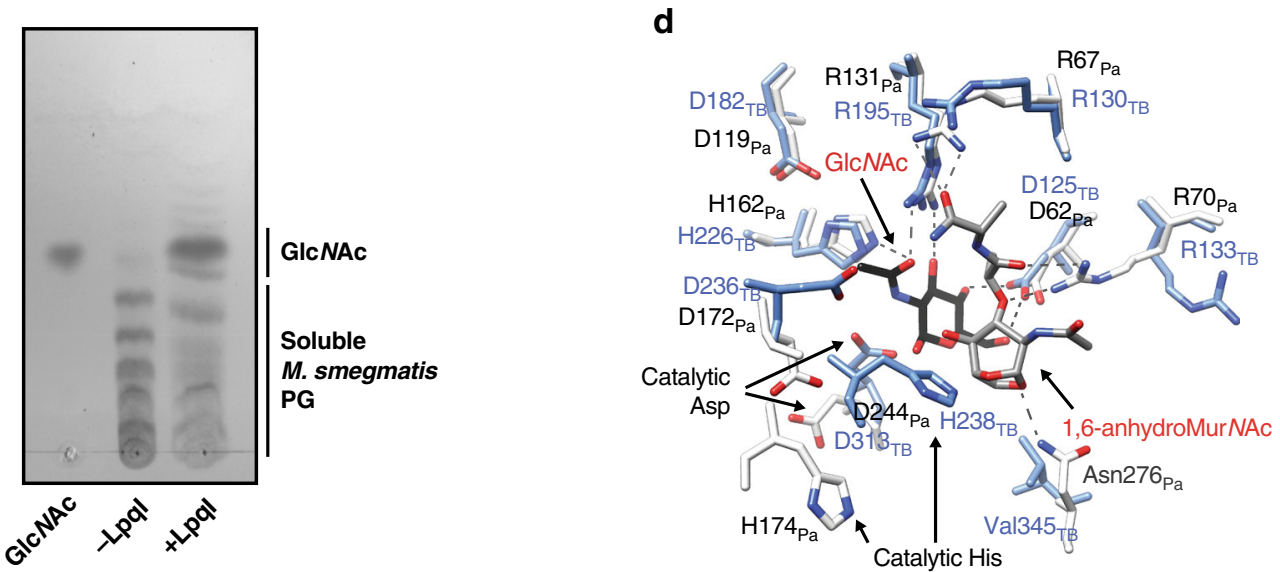

Fig. $2 \mathrm{Lpql}$ is an authentic NagZ-type enzyme. a Reactions including $1 \mu \mathrm{M} \mathrm{Lpql} \mathrm{and} \mathrm{the} \mathrm{indicated} \mathrm{chromogenic} \mathrm{substrates} \mathrm{at} 1 \mathrm{mM}$ were incubated at $37^{\circ} \mathrm{C}$ and release of $p N P$ or $4 \mathrm{MU}$ was followed by absorbance or fluorescence respectively. Cleaveage of $4 \mathrm{MU}-\mathrm{GlcNAc}$ is indicated by blue triangles. $\mathbf{b}$ Lpql was incubated with increasing concentrations of $4 \mathrm{MU}-\mathrm{GlcNAc}$. The rate of $4 \mathrm{MU}$ release was plotted and the curve fit with the Michaelis-Menton equation using GraphPad Prism 7.0. ( $n=3$, error bars are \pm SD). c Thin-layer chromatograph of reaction products showing that Lpql is able to release GlcNAc from soluble muropeptides derived from M. smegmatis $\mathrm{mc}^{2} 155$ PG. d The active site of Lpql is highly conserved as evidenced by the similar positioning of key binding residues observed in the GICNAc, 1,6-anhydroMurNAc complex with NagZ $Z_{\mathrm{Pa}}$ (PDB: 5G3R) the main chains of which have been aligned with an RMSD of $2.3 \AA$ using LSQ KAB ${ }^{57}$

NagZ $_{P a}$ complex with LpqI indicates that the appropriate coordinating residues for MurNAc or 1,6-anhydroMurNAc recognition are intact in LpqI, supporting its role in PGrecycling (Fig. 2d) ${ }^{32}$.

LpqI-catalysed utilisation of PG components by mycobacteria. Having confirmed the in vitro activity of LpqI, we sought to determine the fate of its reaction products, MurNAc and GlcNAc, in growing $M$. bovis BCG. Prior research has shown that most mycobacteria are unable to use GlcNAc as a sole carbon source, with $M$. smegmatis being one of the notable exceptions ${ }^{33}$. Amino acids including D-Ala and D-Glu have previously been shown to serve as nitrogen sources for $M$. tuberculosis $\mathrm{H} 37 \mathrm{Rv}$ but they do not serve as carbon sources ${ }^{34}$. L-Ala has also been shown to not serve as a carbon source for $M$. bovis ${ }^{35}$. To our knowledge, recycling of GlcNAc or MurNAc by mycobacteria has not been reported, nor has recycling been tested for soluble PG fragments. To evaluate this, $M$. bovis BCG was cultured in minimal media supplemented with glycerol $(1 \% \mathrm{v} / \mathrm{v})$ or MurNAc $(0.2 \% \mathrm{w} / \mathrm{v})$ in Sauton's minimal media with constant aeration. As observed in Fig. 3a, M. bovis BCG was able to grow using MurNAc as a sole carbon source. To confirm that this was not a phenotype specific to $M$. bovis BCG we also evaluated the ability of $M$. tuberculosis $\mathrm{H} 37 \mathrm{Rv}$ to grow on the same carbon sources with identical results (Fig. 3b). Intriguingly growth on MurNAc in broth was heavily dependent on the aeration of the culture. In contrast, growth on glycerol was unaffected by this change (Fig. 3c). To further evaluate the potential for mycobacteria to take up GlcNAc but use it for purposes other than central metabolism, we tested the ability of $M$. bovis BCG to incorporate ${ }^{14} \mathrm{C}$ GlcNAc into whole cells. Under different growth conditions (rich medium, carbonpoor medium, aerated cultures, static cultures) we were unable to detect significant amounts of GlcNAc being taken up by M. bovis BCG. In all cases, the c.p.m. of the label in whole cells was less than or equal to unlabelled controls. We conclude from these data that pathogenic mycobacteria are able to utilise MurNAc, but not GlcNAc in an $\mathrm{O}_{2}$-dependent fashion.

Mechanism of MurNAc metabolism. Given its structural similarity to GlcNAc, the ability of M. tuberculosis and M. bovis BCG to grow on MurNAc was surprising and so we evaluated the biochemical processing steps associated with MurNAc utilisation. MurNAc is a combination of GlcNAc and D-lactate joined by an ether linkage. This suggests that the bacterium is either using the GlcNAc moiety for glycolysis, or shunting the lactate derived from MurNAc into the TCA cycle. We tested this by inhibiting glycolysis with 2-deoxyglucose in cultures grown using MurNAc, glucose, and glycerol as sole carbon sources (Supplementary Fig. 6). These data suggested that the pathway of MurNAc utilisation did not require glycolysis and indicated that the lactate 
a

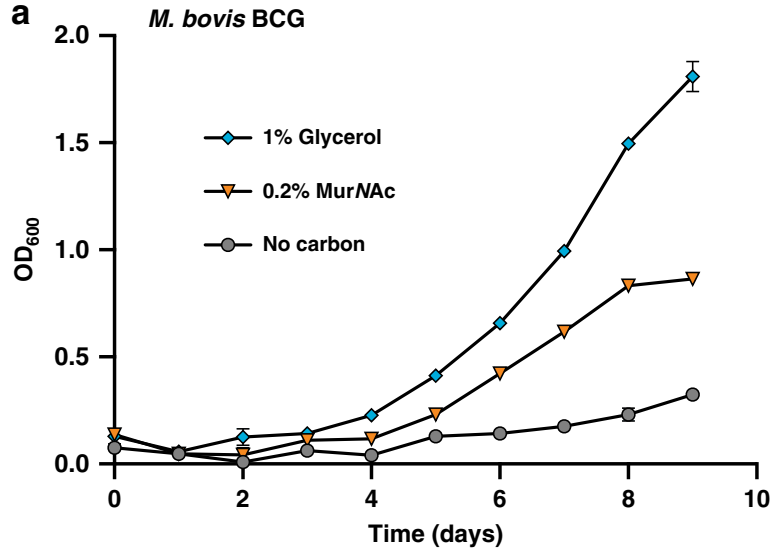

b

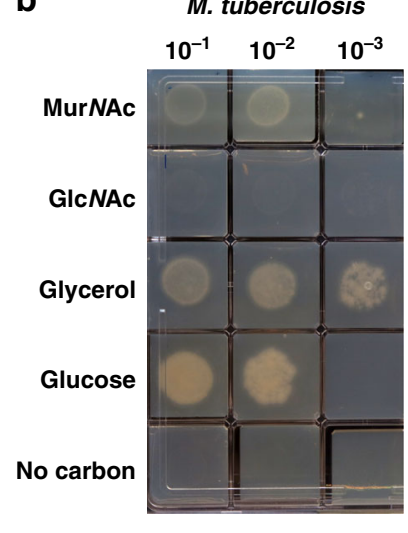

C

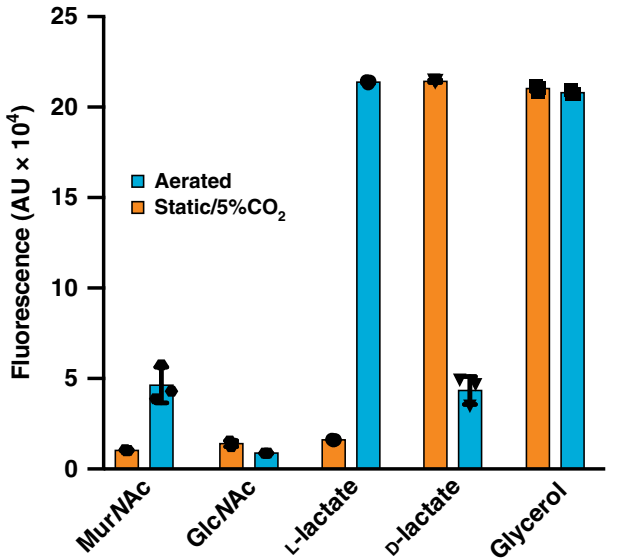

d

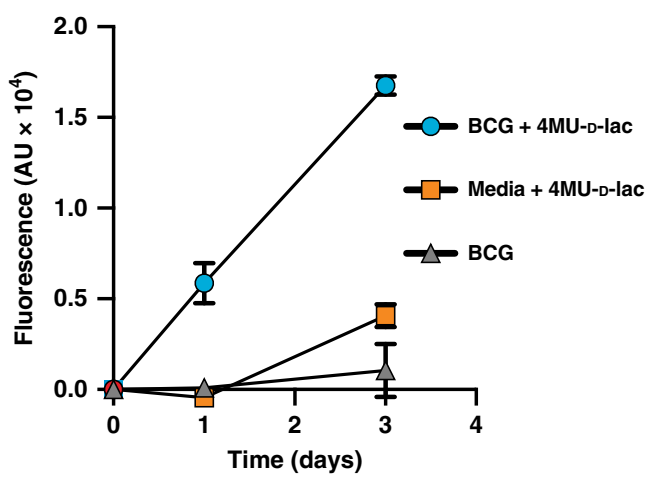

Fig. 3 M. tuberculosis and M. bovis BCG are able to recycle MurNAc. a M. bovis BCG WT was inoculated at a starting OD 600 of 0.1 in Sauton's minimal media containing glycerol (blue diamonds), MurNAc (orange triangles), or no carbon (grey circles) and growth was monitored daily by taking $\mathrm{OD}_{600}$ readings at the indicated time points $(n=3$; error bars are \pm SD). $\mathbf{b}$ M. tuberculosis H37Rv was washed and then serially diluted into fresh carbon-free minimal media. $10 \mu \mathrm{L}$ of each dilution was spotted onto Sauton's agar containing the indicated carbon sources at $5 \mathrm{mM}$. c Growth of M. bovis BCG on $5 \mathrm{mM}$ MurNAc, GICNAC, L-lactate, D-lactate, and glycerol was evaluated in aerated (blue) or static $5 \% \mathrm{CO}_{2}$ (orange) cultures using a resazurin assay ( $n=3$; error bars are $\pm \mathrm{SD}$ ). d Mid-exponential M. bovis BCG was grown in minimal media with $5 \mathrm{mM}$ glycerol including $1 \mathrm{mM} 4 \mathrm{MU}$-D-lactate (blue circles) with constant agitation. At the indicated times, the $4 \mathrm{MU}$ fluorescence of the samples was determined in a BMG Polarstar plate reader $(n=3$; error bars are \pm SD) and compared with controls without cells (orange squares) or without $4 \mathrm{MU}-\mathrm{D}$-lactate (grey triangles)

moiety of MurNAc, was instead most likely serving as a carbon source. Consistent with this, when used as a sole carbon source, growth on L-lactate and MurNAc was $\mathrm{O}_{2}$-dependent, whereas D-lactate was better utilised under static, $5 \% \mathrm{CO}_{2}$ culture conditions, where MurNAc could not be used as a carbon source (Fig. 3c).

These data allow us to hypothesise a mechanism by which $M$. bovis BCG metabolises MurNAc. Given that metabolism of L-lactate and MurNAc are $\mathrm{O}_{2}$-dependent, we anticipate that use of MurNAc follows cleavage of the D-lactate from MurNAc to produce lactate and GlcNAc. In this case, the $\mathrm{O}_{2}$ dependency on MurNAc metabolism is likely the result of an $\mathrm{O}_{2}$-dependent lactate dehydrogenase. Consistent with this, two $\mathrm{O}_{2}$-dependent $\mathrm{L}$ lactate dehydrogenases have been identified in M. tuberculosis (Rv0694, Rv1872c), which are required for growth on L-lactate as a sole carbon source ${ }^{36}$. Given the unusual nature of MurNAc, generation of free lactate by the bacterium would require the activity of a specific lactyl-etherase. To test for the presence of this activity in whole cells, we synthesised a $4 \mathrm{MU}$-D-lactate derivative to serve as a reporter-analogue of MurNAc (Supplementary Fig. 7). Consistent with the presence of a lactyl-etherase, cultures of $M$. bovis BCG were able to release $4 \mathrm{MU}$ from this compound during the course of growth (Fig. 3d). Together these data support a model where mycobacteria cleave the lactyl moiety from MurNAc by an as-yet unidentified enzyme and utilise the product of that reaction as a carbon source under aerated conditions.

LpqI-dependent uptake of PG metabolites by mycobacteria. Although our data strongly support metabolism of MurNAc by M. bovis BCG, we wanted to confirm the role of LpqI in mycobacterial PG-recycling. To do this we constructed a mutant strain of $M$. bovis BCG-lacking lpqI using specialised transduction ${ }^{37}$. To validate that LpqI is the only $\beta-N$-acetylglucosaminidase produced by $M$. bovis BCG, we used a whole-cell $\beta$-N-acetylglucosaminidase assay. This demonstrated that $M$. bovis BCG $\Delta l p q i$ is devoid of $\beta-N$-acetylglucosaminidase activity as the amount of $4 \mathrm{MU}$ released is not significantly different from the spontaneous release in sterile media (Fig. 4a). This deficiency is complemented by re-introduction of the lpqI gene at a distal chromosomal location under the control of its native promoter $(\Delta l p q I:: l p q I)$ and is not complemented by the empty vector $(\Delta l p q I:: \mathrm{EV})$ (Fig. 4a). Growth of $\Delta l p q I$ in vitro is also unaltered as compared to the wild-type (Fig. $4 \mathrm{~b}$ ). This mutant therefore provided us with an opportunity to probe the role of disaccharide cleavage in mycobacterial PG-recycling.

The order in which muropeptides are recycled, and the chemical structure of the recycled material is critical for the 

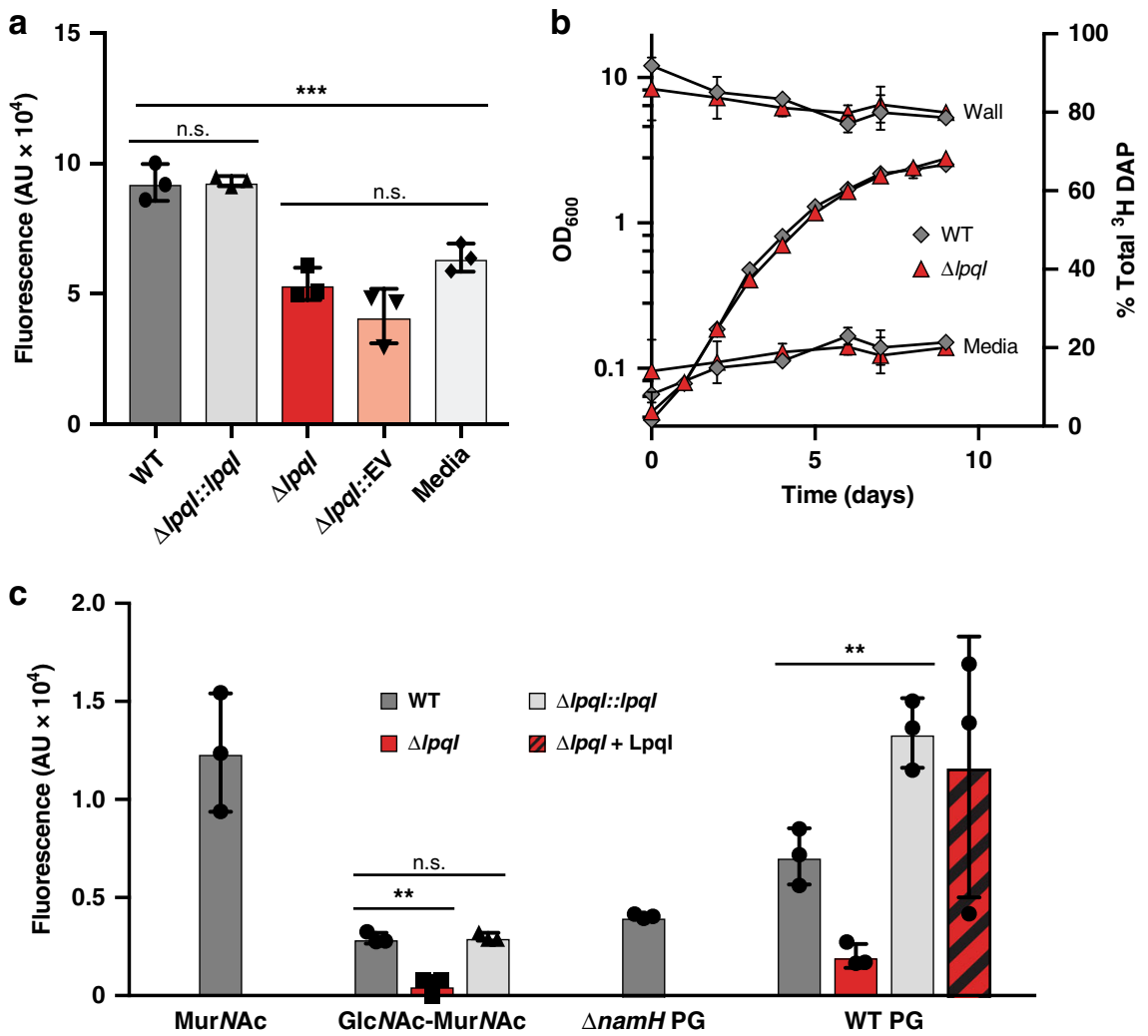

Fig. $4 \mathrm{M}$. bovis BCG is able to recycle PG. a M. bovis BCG WT, $\Delta / p q l, \Delta / p q l:: / p q l$ and $\Delta / p q l::$ Empty Vector were incubated with $1 \mathrm{mM} 4 \mathrm{MU}-\mathrm{GlcNAc}$ in minimal media. After 3 days the fluorescence of the cultures were measured $(n=3$; error bars are \pm SD). b M. bovis BCG WT (grey diamonds) and $\Delta / p q l$ (red triangles) were simultaneously evaluated for release of cell wall peptides and growth ( $n=3$; error bars are \pm SD). c M. bovis BCG WT (dark grey bars), $\Delta / p q l$ (red bars), and $\Delta / p q l:: 1 p q l$ (light grey bars) were evaluated for their growth using MurNAc ( $5 \mathrm{mM})$, GlcNAc-MurNAc (2 mM), soluble PG from M. smegmatis $\mathrm{mc}^{2} 155$ wild-type or $\triangle \mathrm{namH}$ ( 5 and $2.5 \mathrm{mM}$, respectively), and soluble PG, which had been pre-digested wth Lpql (striped bars) as sole carbon sources using a resazurin assay $\left(n=3 ;{ }^{\star \star \star}=p<0.001 ;{ }^{\star \star}=p<0.005\right)$. Statistical significance determined using a two-tailed $t$-test in GraphPad Prism 7

immune sensing of the bacterium. To determine the order of PGrecycling steps, we first assessed the impact of the loss of lpqI on the recycling of cell wall material. To investigate this, we generated radio-labelled muropeptides and tested them in whole-cell uptake assays. Radio-labelled muropeptides had to be generated in $M$. smegmatis $\mathrm{mc}^{2} 155$ owing to the inability of $M$. bovis $\mathrm{BCG}$ to take up ${ }^{14} \mathrm{C}$ GlcNAc under the conditions we tested. As shown in Supplementary Fig. 8, the M. bovis BCG $\Delta l p q I$ mutant took up $\sim 25 \%$ less of the labelled PG than the wild-type $(\sim 3 \%$ vs. $\sim 4 \%$, respectively). In a similar assay using PG fragments labelled with ${ }^{3} \mathrm{H}$ diaminopimelic acid (DAP) the mutant did take up slightly less of the PG than the wild-type, however this difference was not found to be significant using a two-tailed $t$ test (Supplementary Fig. 8). To probe this result further, we pre-labelled cells with ${ }^{3} \mathrm{H}$ DAP and monitored the release of the label into the culture media. Supporting the data above, we observed no significant differences between the wildtype and the $\Delta l p q I$ strain with respect to the amount of label released to the media (Fig. 4b). From these experiments, we concluded that LpqI is involved in amino sugar recovery but is not required for stem-peptide recycling.

Given the inability of $M$. bovis BCG to take up radiolabeled GlcNAc we were unable to repeat the previous experiment using ${ }^{14} \mathrm{C}$ GlcNAc and follow release of this sugar to the media. To unambiguously test the impact of deleting lpqI on amino sugar recycling by the bacterium, we evaluated its ability to grow on MurNAc, GlcNAc-MurNAc and PG from both wild-type $M$. smegmatis $\mathrm{mc}^{2} 155$ and $\Delta$ namH strain, which lacks the characteristic $\mathrm{N}$-glycolyl modification of MurNAc (Fig. 4c). The $\Delta l p q I$ strain was not deficient for growth on MurNAc, however unlike the wild-type strain it was unable to grow on GlcNAc-MurNAc and exhibited only very weak growth on soluble PG as a sole carbon source (Fig. 4c). This growth was rescued by pre-digestion of the muropeptides with LpqI (Fig. 4c). Together these data indicate that $l p q I$ is required for PG-derived amino sugar recycling in $M$. bovis BCG.

Phenotypic characterisation of a $\Delta l p q I$ mutant. As indicated above, loss of LpqI did not alter the growth rate of the bacterium in vitro. However, given that NagZ-like proteins have been found to play a role in $\beta$-lactam sensitivity in other bacteria we sought to determine the antimicrobial sensitivity of the $\Delta l p q I$ strain. In contrast to inhibition of $P$. aeruginosa NagZ, deletion of $l p q I$ resulted in an increase in survival in the presence of lysozyme and all cell wall active antibiotics tested (Fig. 5a-d) ${ }^{38}$. A smaller impact on survival in the presence of the protein synthesis inhibitor chloramphenicol was observed (Fig. 5e). This increase in resistance is not owing to a change in cell wall permeability as determined by ethidium bromide uptake (Fig. 5f). These data indicate that lpqI-dependent amino sugar recycling is involved in the expression of antibiotic and lysozyme resistance by mycobacteria in vitro.

\section{Discussion}

In an attempt to develop diagnostic media for the identification of mycobacteria, several groups in the 1960s observed that $M$. tuberculosis and most other mycobacteria could not metabolise GlcNAc as a sole carbon source ${ }^{33,34}$. This, along with the absence 

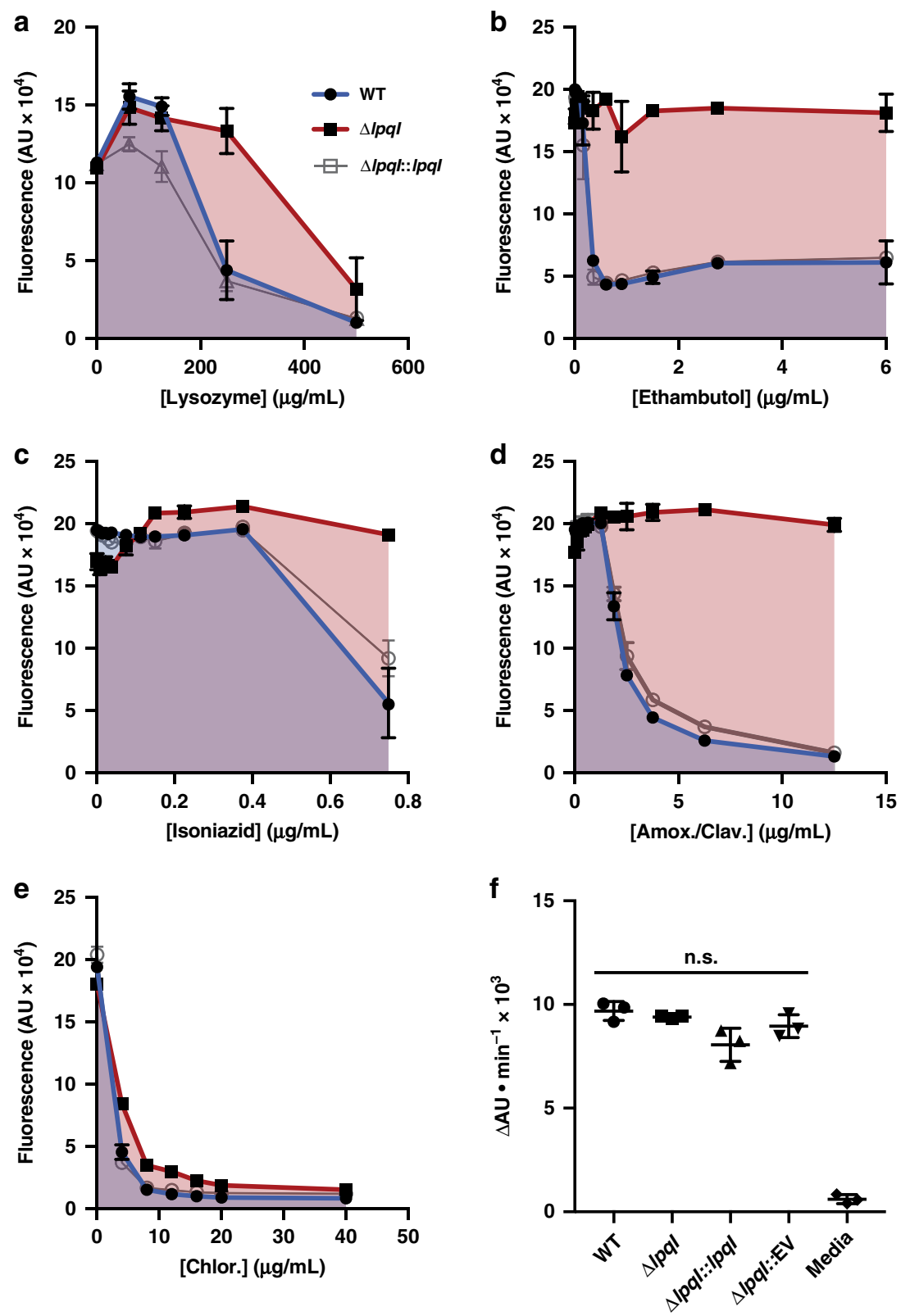

Fig. 5 Loss of Lpql leads to lysozyme and antibiotic resistance. a-e M. bovis BCG WT (blue), $\Delta / p q l$ (red), and $\Delta /$ pql::lpql (grey) were incubated with increasing concentrations of lysozyme or antibiotics at a starting $\mathrm{OD}_{600}$ of 0.1 . After 7 days incubation total growth was assessed using a resazurin assay, where total fluorescence correlates with respiration and growth $(n=3$; error bars are \pm SD; Amox./Clav-amoxicillin plus clavulanic acid; Chlor.chloramphenicol). $\mathbf{f} M$. bovis BCG WT, $\Delta / p q l$ and $\Delta / p q l:: / p q l$ and $\Delta / p q l:: E V$ were incubated with $\mathrm{EtBr}$ and the rate of EtBr uptake was monitored as an increase in fluorescence. No significant differences were found in pairwise $t$ tests across all strains $(n=3$; error bars are \pm SD). Statistical significance determined using a two-tailed $t$ test

of known PG recycling-associated genes lead to the assumption that $\mathrm{PG}$ recycling is absent in pathogenic mycobacteria. Based on our data and the literature, it is clear that not only is M. tuberculosis able to recycle its PG, it is generating two distinct classes of molecules. These are GlcNAc-MurNAc-peptide, which is sensed by the host, and GlcNAc-MurNAc that is sensed by the bacterium. Although host-sensing of PG is unaffected by the presence of GlcNAc on NOD-stimulatory molecules, our data indicate that LpqI acts as a regulator for GlcNAc-MurNAc levels by cleaving disaccharides and allowing the breakdown of MurNAc. In other bacteria, cell wall damage can trigger various stress responses, and so it is likely that a build-up of GlcNAc-MurNAc disaccharides may trigger a stress-like response in mycobacteria ${ }^{39}$. Consistent with this, lpqI is encoded adjacent to a universal stress response protein in several species of mycobacteria (Supplementary Fig. 1).

As a starting point to investigate PG recycling in $M$. tuberculosis, we characterised the core mycobacterial protein, LpqI. Despite the absence of other known PG-recycling proteins, we have shown that LpqI is an authentic exo-acting $\beta$-N-acetylglucosaminidase, which is able to cleave PG fragments in vitro. Consistent with a role in PG-recycling, M. bovis BCG $\Delta l p q I$ is unable to grow on soluble PG as a sole carbon source, whereas recycling of the stem-peptide is unaltered in this mutant. Together, our data demonstrate that M. bovis BCG and M. tuberculosis remove the stem-peptide from $P G$ fragments prior to disaccharide cleavage and lactyl-ether removal (Fig. 6). The 


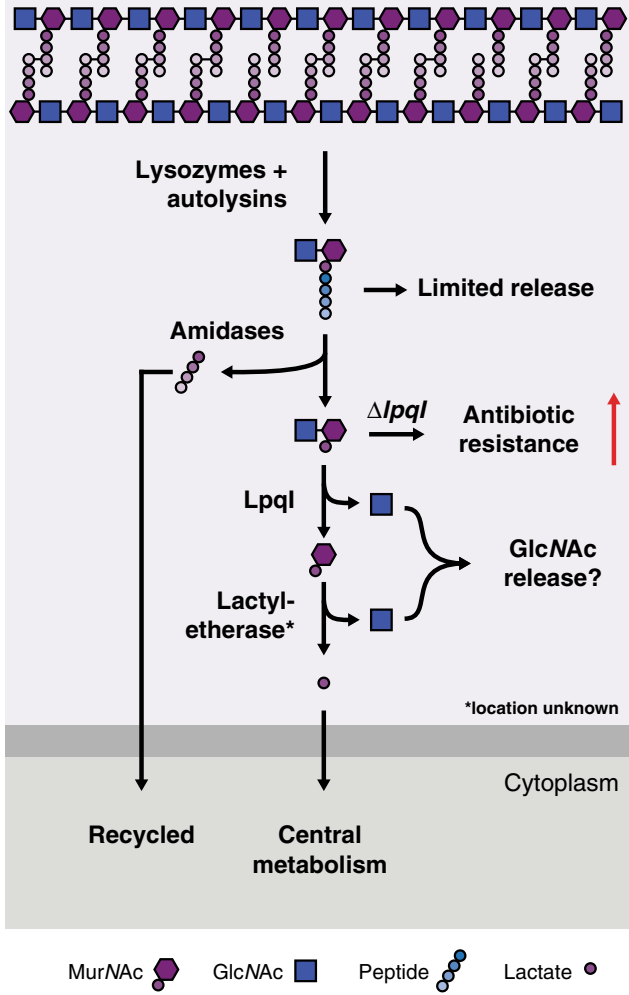

Fig. 6 Peptidoglycan recovery pathway in pathogenic mycobacteria. Based on our observations we can propose the following model for PG recycling and recovery in mycobacteria. Cleavage of the cell wall by endogenous autolysins or host-derived lysozyme generates muropeptides. Some of this material undergoes limited release to stimulate the host immune system. The remainder are subsequently degraded by amidases and other peptidases. Lpql then cleaves GlcNAc-MurNAc, which is followed by D-lactyl-ether cleavage. Lactate can then be used by the cell under aerobic conditions and GICNAC (or its derivatives) are most likely released. Perturbation of this system by deleting Lpql leads to increased resistance to anti-mycobacterial agents

processing of GlcNAc-MurNAc by LpqI prior to lactyl-ether cleavage is also supported by our LpqI crystal structure in which the lactate-binding residue R67 from the $P$. aeruginosa structure is conserved (LpqI: R130), suggesting that the physiological substrate of this enzyme possesses the lactyl group ${ }^{32}$.

Our sole carbon source assays indicate that although the bacteria are unable to metabolise GlcNAc, surprisingly they can use MurNAc as a sole carbon source (Fig. 3). This is despite the fact that they lack an ortholog of the only known lactyl-etherase, MurQ that cleaves an otherwise stable lactyl-ether in the cytoplasm of most model organisms (Fig. 1). Our data indicate that rather than using the GlcNAc portion of the MurNAc, the bacteria are cleaving the lactyl-ether and metabolising the liberated lactate. During our study we found that M. bovis BCG was only able to grow on MurNAc under aerated conditions. This was also found to be the case for L- but not D-lactate, which served as a much better carbon source under $\mathrm{O}_{2}$ - limiting conditions. This suggests that L-lactate is likely the metabolised product of this reaction. The presence of a specific lactyl-etherase is further supported by the turnover of a $4 \mathrm{MU}$-D-lactate reporter compound by $M$. bovis BCG. The $\mathrm{O}_{2}$ dependence of growth on MurNAc is intriguing as $\mathrm{N}$-glycolylation is also an $\mathrm{O}_{2}$-dependent activity, suggesting significant alterations to PG metabolism in hypoxic vs. aerobically growing mycobacteria ${ }^{40}$. Consistent with this observation, expression of $l p q I$ is upregulated twofold during re-aeration after re-activation from non-replicating persistence in the Wayne hypoxia model $^{41}$.

The fate of GlcNAc in this pathway remains unclear, although our data and prior observations suggest that the bacteria do not re-use this sugar. The inability to utilise this sugar is surprising given the conservation of the nagA (Rv3332) gene in mycobacteria $^{42}$. However, it is possible that an alternative GlcNAcrecovery pathway exists that was not functional under the conditions we have tested. This is hinted at, with our ${ }^{14} \mathrm{C}$-labelled muropeptides, where incorporation of the labelled-GlcNAc is not expected, given the lack of monomeric GlcNAc utilisation by the cells. During the production of radio-labelled PG in M. smegmatis, a portion of the GlcNAc will have been used by the bacterium to generate MurNAc rather than strict shunting of GlcNAc into UDP-GlcNAc for cell wall biosynthesis. The subsequent removal of the lactyl-ether from this ${ }^{14} \mathrm{C}$-MurNAc by $M$. bovis BCG would then follow steps and intermediates we do not yet fully understand. Indeed, bacterial etherases comprise a diverse number of mechanisms and potential reaction products and so a product other than free GlcNAc is entirely possible ${ }^{43}$. We are currently trying to identify and characterise the enzyme responsible for the observed lactyl-etherase activity.

In conclusion, we have identified a PG recovery pathway in pathogenic mycobacteria. We have shown that this occurs in a step-wise fashion by removing stem-peptide from PG and subsequently cleaving the PG-disaccharide and finally releasing the D-lactate from free MurNAc. Finally, we have shown that recycling of PG by these bacteria is important for lysozyme and antibiotic resistance.

\section{Methods}

Bacterial strains and growth conditions. Unless stated otherwise, all chemicals and reagents were purchased from Sigma Aldrich. M. tuberculosis H37Rv, M. bovis BCG (Pasteur), and related mutants were maintained on Middlebrook 7H10 agar or $7 \mathrm{H} 9$ broth supplemented with $10 \%$ OADC enrichment and $0.05 \%$ Tween 80 . Appropriate kanamycin or hygromycin was added at 25 or $50 \mu \mathrm{g} \mathrm{mL}^{-1}$, respectively. Mycobacterium smegmatis strains $\mathrm{mc}^{2} 155$ and $\mathrm{mc}^{2} 155 \Delta$ namH were maintained on Tryptic Soy Broth or Tryptic Soy Agar where appropriate ${ }^{40}$. For growth on defined carbon sources, strains were cultivated in Sauton's minimal medium containing per $1 \mathrm{~L}, 4 \mathrm{~g}$ asparagine, $2 \mathrm{~g}$ citric acid, $0.5 \mathrm{~g} \mathrm{~K} \mathrm{HPO}_{4}, 0.5 \mathrm{~g}$ $\mathrm{MgSO}_{4} * 7 \mathrm{H}_{2} \mathrm{O}$, and $0.05 \mathrm{~g}$ ferric ammonium citrate, $0.05 \%$ tyloxopol and $5 \mathrm{mM}$ of each carbon source unless stated otherwise ${ }^{44}$. E. coli strains were grown in lysogeny broth and supplemented with kanamycin at $50 \mu \mathrm{g} \mathrm{mL}^{-1}$ or hygromycin at $150 \mu \mathrm{g} \mathrm{mL}^{-1}$ where appropriate. GlcNAc-MurNAc was purchased from Toronto Research Chemicals.

Cloning and purification of $\mathbf{L p q}_{\mathbf{T B}}$. The gene encoding LpqI (Rv0237) was amplified from $M$. tuberculosis H37Rv genomic DNA using standard PCR conditions with the lpqITBSUMOF and lpqITBSUMOR primers and cloned into the TA site of the Champion pET-SUMO expression plasmid (Invitrogen) according to the manufacturer's specifications (all primers are found in Supplementary Table 1). For production of LpqI $1 \mathrm{~L}$ of E. coli BL21 [pLpqITB] grown in Terrific Broth to an $\mathrm{OD}_{600}$ of 0.6 , chilled to $20^{\circ} \mathrm{C}$ and induced with $1 \mathrm{mM}$ IPTG and grown for a further $18 \mathrm{~h}$ before being collected by centrifugation. Cells were resuspended in $25 \mathrm{mM}$ Tris- $\mathrm{HCl}, 300 \mathrm{mM} \mathrm{NaCl}, 10 \mathrm{mM}$ imidazole $\mathrm{pH} 7.8$ and lysed via three passages through a French pressure cell. The protein was purified using standard IMAC procedures with washes of lysis buffer, lysis buffer including $50 \mathrm{mM}$ imidazole and finally eluted with $500 \mathrm{mM}$ imidazole in lysis buffer. Eluted protein was dialysed exhaustively against $25 \mathrm{mM}$ Bis-Tris, $100 \mathrm{mM} \mathrm{NaCl} \mathrm{pH} 7.8$ in the presence of recombinant Ulp1 protease, which specifically cleaves the $\mathrm{His}_{6}$-SUMO tag. Digested protein was passed through a second IMAC column (1 mL HisTrap FF, GE Healthcare) and the flow-through fraction was found to contain pure, untagged LpqI. Purified protein was dialysed into $25 \mathrm{mM}$ Bis-Tris $\mathrm{pH} 6.5$, $100 \mathrm{mM} \mathrm{NaCl}$.

Cloning and purification of $\mathbf{R p f B}$. RpfB was cloned according to the same procedure as LpqI using the primers RpfBFwd and RpfBRev. Expression and purification was achieved using the same general procedure as LpqI.

Crystallography. Prior to crystallisation, LpqI was concentrated to $20 \mathrm{mg} \mathrm{mL}^{-1}$ in $25 \mathrm{mM}$ Bis-Tris pH 7.5, $100 \mathrm{mM} \mathrm{NaCl}$. LpqI crystals were grown by the sittingdrop vapour diffusion method by mixing an equal volume of protein solution with 
1.1 м sodium malonate, $0.1 \mathrm{~m}$ HEPES, $0.5 \% \mathrm{w} / \mathrm{v}$ Jeffamine ED-2001 (pH 7.0). Crystals were cryo-protected with a saturated solution of sodium malonate and flash-cooled in liquid nitrogen. X-ray data were collected at beamline I04-1 of the Diamond Light Source, Oxford. Data were auto-processed using Xia2 and general file manipulations were performed using the CCP4 suite of programmes ${ }^{45,46}$. The structure was phased by molecular replacement using the unpublished M. smegmatis LpqI structure (PDB: 4YYF, 67\% sequence identity) using the programme PHASER (TFZ of 17). The structure was subsequently auto-built in PHENIX and the remaining parts were built in COOT with further refinement using PHENIX and PDB-REDO ${ }^{47-49}$

Kinetic characterisation of Lpql. Purified LpqI was evaluated for glycoside hydrolase activity using a variety of substrates. As an initial screening assay, Lpq was incubated at $1 \mu \mathrm{M}$ with either 4-methylumbeliferyl (4MU) or $p$-nitrophenyl $(p N P)$ derivatives of a variety of sugars (arabinose, galactose, galactosamine, arabinofuranose, glucose, mannose, mannosamine, glucosamine, and $\mathrm{N}$-acetylglucosamine) in Bis-Tris $\mathrm{pH} 7.5,100 \mathrm{mM} \mathrm{NaCl}$ at $37^{\circ} \mathrm{C}$. The release of $p \mathrm{NP}$ was followed by change in absorbance at $420 \mathrm{~nm}$, whereas production of 4menthylumbelliferone was monitored by fluorescence (Ex. $355 \mathrm{~nm}$; Em $460 \mathrm{~nm}$ ) in a BMG Polarstar spectrophotometer. Kinetic characterisation of $\mathrm{LpqI}_{\mathrm{TB}}$ was conducted using varying concentrations of $4 \mathrm{MU}-\mathrm{Gl}$ cNAc. The raw data were compared to standards of $p \mathrm{NP}$ or $4 \mathrm{MU}$. All data were analysed using GraphPad Prism 7.

Substrate specificity of LpqI. To determine whether LpqI was an endo- or exoacting enzyme we incubated $0.1 \mu \mathrm{M}$ of either LpqI or ChitB (Generon) with $5 \mathrm{mM}$ $\mathrm{N}, \mathrm{N}^{\prime}, \mathrm{N}^{\prime \prime}, \mathrm{N}^{\prime \prime}, \mathrm{N}^{\prime \prime}{ }^{\prime}, \mathrm{N}^{\prime \prime}$ "' '-hexacetylchitohexaose $\left(\mathrm{GlcNAc}_{6}\right)$ in $25 \mathrm{mM}$ phosphate buffer pH 6.5. After $3 \mathrm{~h}, 30 \mu \mathrm{L}$ the sample was reduced with $10 \mathrm{mg} \mathrm{mL}^{-1}$ (final) $\mathrm{NaBH}_{4}$ for $30 \mathrm{~min}$. The $\mathrm{NaBH}_{4}$ was quenched with $20 \%$ phosphoric acid and the $\mathrm{pH}$ reduced to $\sim 4$. Reaction products were separated by HPLC on a Dionex UPLC system with a Thermo Hypercarb column $(100 \times 2.1 \mathrm{~mm})$. Separation was achieved with a linear gradient from $0.1 \%$ trifluoroacetic acid (TFA) in $\mathrm{H}_{2} \mathrm{O}$ to $30 \%$ acetonitrile, $0.1 \%$ TFA over 15 min and peaks were detected with absorbance at $210 \mathrm{~nm}$. Peaks were identified using standards of known length. To determine whether LpqI could act as an autolysin and cleave insoluble mycobacterial PG we labelled insoluble PG from M. smegmatis $\mathrm{mc}^{2} 155$ with fluorescein isothiocyanate ${ }^{50}$. LpqI, mutanolysin or a chitinase were incubated at a final concentration of $1 \mu \mathrm{M}$ overnight with $1 \mathrm{mg} \mathrm{mL}^{-1}$ FITC-PG conjugate in $25 \mathrm{mM}$ sodium phosphate buffer $\mathrm{pH}$ 6.5. The sample was then filtered and the fluorescence of the soluble fraction was read (Ex. 495, Em, 519) in a BMG Fluorostar plate reader.

Digestion of cell wall material with mutanolysin or RpfB was carried out overnight at $37^{\circ} \mathrm{C}$ in $20 \mathrm{mM}$ ammonium acetate buffer ( $\mathrm{pH}$ 6.5) with continuous mixing. Following digestion, solubilised muropeptides were isolated using graphitised carbon solid-phase-extraction cartridges ${ }^{51}$. Purified fractions were evaporated to dryness and the concentration of reducing sugars in the pool of soluble muropeptides was assessed using the 3-methyl-2-benzothiazolinone hydrazone assay ${ }^{52}$. For RpfB-generated muropeptides the concentration was estimated gravimetrically using an average molecular weight of $953.41 \mathrm{Da}$ for GlcNAc-1,6-anhydroMurNGlyc-tetrapeptide.

Reactions including $1 \mu \mathrm{M} \mathrm{LpqI} \mathrm{I}_{\mathrm{TB}}$, and $0.5 \mathrm{mM}$ mutanolysin-derived PG fragments or $0.1 \mathrm{mM}$ RpfB-derived PG fragments in $25 \mathrm{mM}$ ammonium acetate buffer $\mathrm{pH} 6.5$ were incubated for $18 \mathrm{~h}$ at $37^{\circ} \mathrm{C}$. The reactions were then evaluated by TLC (Silica $60 \mathrm{~F}_{254}$, Merck, Germany) using a mobile phase consisting of 1-butanol, methanol, ammonium hydroxide and water at a ratio of 5:4:2:1. TLCs were stained with $\alpha$-naphthol and developed by charring.

Mutant generation. To generate the $\Delta l p q I$ strain we used specialised transduction according to established protocols ${ }^{37}$. A recombinant $l p q I$ knockout phage was designed to replace the chromosomal lpqI gene using homologous flanking regions to $l p q I$ with a $s a c B$ gene and a hygromycin resistance cassette in-between using the LL-lpqIBCG, LR-lpqIBCG, RR-lpqIBCG, and RL-lpqIBCG primers. The resulting phage was transduced into $M$. bovis BCG and transductants were selected on $7 \mathrm{H} 10$ agar plates containing $75 \mu \mathrm{g} \mathrm{mL}^{-1}$ hygromycin. The mutant was verified by PCR and phenotypically with $4 \mathrm{MU}-$ GlcNAc where loss of $l p q I$ was expected to abolish turnover of this fluorescent substrate. The complemented strain was generated by incorporating the lpqI gene and $150 \mathrm{bp} 5^{\prime}$ to the start codon containing the promoter sequence into the promoter-less integrative mycobacterial shuttle vector pMV306 using primers lpqIBCGCompF and lpqIBCGCompR to generate the resulting pMV306-lpqI plasmid ${ }^{53}$. This was electroporated into M. bovis BCG $\Delta l p q I$ and transformants were selected on $7 \mathrm{H} 10$ agar containing hygromycin and kanamycin. The complemented mutant was verified phenotypically with $4 \mathrm{MU}-$ GlcNAc. A control strain was also generated using the empty pMV306 vector using the same protocols.

Antimicrobial testing. Mid-exponential cultures of M. bovis BCG and derivative strains were diluted to $\mathrm{OD}_{600}=0.1$ in fresh $7 \mathrm{H} 9$ media. $100 \mu \mathrm{L}$ of this culture was added to a 96-well plate with the addition of $1 \mu \mathrm{L}$ of antibiotic/lysozyme to achieve the desired final concentration as indicated. These were incubated for 7 days at $37^{\circ} \mathrm{C}$ at which point $30 \mu \mathrm{L}$ of $0.02 \% \mathrm{w} / \mathrm{v}$ resazurin and $12.5 \mu \mathrm{L}$ of $20 \%$ Tween $80 \mathrm{v} / \mathrm{v}$ was added to the culture. This was incubated overnight at $37^{\circ} \mathrm{C}$ and the production of resorufin was determined by fluorescence (Ex. $530 \mathrm{~nm}, \mathrm{Em} .590 \mathrm{~nm}$ ) using a BMG Polarstar plate reader.

Rapid purification of mycobacterial cell wall. Rapid purification of cell wall from small cultures was carried out using a modified phenol extraction protocolli ${ }^{19}$ Mycobacterial cells were grown to mid-exponential phase and collected by centrifugation. These were washed with cold phosphate-buffered saline (PBS) and resuspended in PBS and the cells were lysed in a Percellys Evolution Bead Beater at $5000 \mathrm{rpm}$ for $3 \mathrm{~min}$. The lysate was then transferred to glass culture tubes to which $2 \mathrm{~mL}$ of $98 \%$ phenol was added and vortexed for $1 \mathrm{~min}$. This was heated for $1 \mathrm{~h}$ at $70{ }^{\circ} \mathrm{C}$, allowed to cool and the insoluble material was collected by centrifugation at $3220 \times g$. The aqueous phase was removed and $4 \mathrm{~mL}$ of methanol was added. This was vortexed and centrifuged again. Finally, the pellet was washed three times with methanol and once with water before being frozen or used for subsequent enzymatic treatment.

Large-scale purification of PG. Purification of PG from M. smegmatis was achieved following established protocols ${ }^{54}$. Six liters of $M$. smegmatis were grown with aeration to mid-exponential phase $\left(\mathrm{OD}_{600}=\sim 0.6\right)$ at which point they were harvested by centrifugation, resuspended in a minimal volume of PBS and lysed by sonication. The resulting lysate was brought to $4 \%$ sodium dodecyl sulphate (SDS) and boiled under reflux for $3 \mathrm{~h}$. The insoluble material was collected by centrifugation and washed with water until the SDS was completely removed (at least 7 washes) to yield mycolyl-arabinogalactan-peptidoglycan (mAGP). The mAGP was incubated for 4 days in $0.5 \%(\mathrm{w} / \mathrm{v}) \mathrm{KOH}$ in methanol at $37^{\circ} \mathrm{C}$ before being washed three times with methanol. The mycolic acids were extracted with three washes of diethyl ether. The phosphodiester linking the AG-PG complex was cleaved using $0.2 \mathrm{M} \mathrm{H}_{2} \mathrm{SO}_{4}$ and the PG was separated from the solubilized AG by centrifugation prior to neutralisation with $\mathrm{NaCO}_{3}$ and washed with water three times. The insoluble PG pellet was sequentially digested with $\alpha$-amylase $\left(100 \mu \mathrm{g} \mathrm{mL}{ }^{-1}\right)$, DNase $\left(10 \mu \mathrm{g} \mathrm{mL}^{-1}\right)$ and RNase $\left(5 \mu \mathrm{g} \mathrm{m}^{-1}\right)$ for $8 \mathrm{~h}$ before proteinase $\mathrm{K}\left(100 \mu \mathrm{g} \mathrm{mL}^{-1}\right)$ digestion overnight at $37^{\circ} \mathrm{C}$. The $\mathrm{PG}$ pellet was resuspended in a minimal volume of $1 \%$ SDS and boiled under reflux for $3 \mathrm{~h}$ before the SDS was removed by centrifugation and washing with water (at least seven times). The resulting material was lyophilised and stored at $-20^{\circ} \mathrm{C}$ until it was needed. Digestion and quantitation of this material was carried out as described above.

Synthesis of 4MU-D-lactate. Instead of the 2- or 3- step protocols published for the synthesis of $4 \mathrm{MU}-\mathrm{D}$-lactate previously, we used a simplified one step method $^{55,56}$. In total, $1.5 \mathrm{~g}$ of (s)-(-)-bromopropionic acid was added to $1 \mathrm{~g}$ of 4-methylumbelliferone stirring in $40 \mathrm{~mL}$ anhydrous dimethylformamide and $0.75 \mathrm{~g}$ $\mathrm{Cs}_{2} \mathrm{CO}_{3}$. This was stirred at room temperature overnight and the product was extracted three times with water/ethyl-acetate and the organic phase was dried over sodium sulphate. The organic phase was then filtered and evaporated to dryness. The product was subsequently purified using silica chromatography and was dried as a crystalline white solid.

Turnover of 4MU reporter compounds by $\boldsymbol{M}$. bovis BCG. To test turnover of $4 \mathrm{MU}-\mathrm{GlcNAc}$ or $4 \mathrm{MU}-\mathrm{D}-\mathrm{lactate}$ by whole cells, $100 \mu \mathrm{L}$ of a mid-exponential culture $\left(\mathrm{OD}_{600}=0.6\right)$ was added to a sterile 96-well plate in Sauton's minimal media supplemented with $0.05 \%$ Tween and $1 \%$ glycerol in addition to $1 \mathrm{mM} 4 \mathrm{MU}-\mathrm{D}-$ lactate or $4 \mathrm{MU}$-GlcNAc. Similar controls lacking cells or the reporter compound were included as well. This was incubated at $37^{\circ} \mathrm{C}$ and mixed at $300 \mathrm{rpm}$. On the indicated days the $4 \mathrm{MU}$ fluorescence of the sample was read on a BMC PolarStar microplate reader with a constant gain setting.

Turnover of $\boldsymbol{M}$. bovis BCG PG in vitro. Cultures of $M$. bovis BCG wild-type, $\Delta l p q I$, and $\Delta l p q I:: l p q I$ were grown to an $\mathrm{OD}_{600}$ of 0.6 in the presence of $10 \mu \mathrm{Ci}{ }^{3} \mathrm{H}$ mesoDAP, at which point they were collected by centrifugation, washed three times with sterile media and diluted to 0.01 in fresh culture flasks. Periodically a sample of $0.5 \mathrm{~mL}$ was taken, and the cells were collected by centrifugation. The spent medium was mixed with $10 \mathrm{~mL}$ scintillation fluid and counted using a liquid scintillation counter The cell pellet was resuspended in $10 \%$ SDS, boiled for $20 \mathrm{~min}$, and centrifuged again. The cell wall material was then resuspended in $1 \mathrm{~mL}$ scintillation fluid and the material was counted in a liquid scintillation counter. The counts of the cell wall and the media were added together to give total ${ }^{3} \mathrm{H}$ DAP in each culture and the data is presented as a percentage of that total. During the course of the experiment the $\mathrm{OD}_{600}$ of the culture was monitored daily. All measurements are from three biological replicates.

Reporting summary. Further information on research design is available in the Nature Research Reporting Summary linked to this article.

\section{Data availability}

The atomic coordinates and structure factors of LpqI are available from Protein Data Bank with accession code 6GFV. All data used to generate the figures are available in the Source Data file. Other data that support the findings of this study are available from the authors on request. 
Received: 2 July 2018 Accepted: 14 May 2019

Published online: 14 June 2019

\section{References}

1. Jankute, M., Cox, J. A. G., Harrison, J. \& Besra, G. S. Assembly of the Mycobacterial cell wall. Annu Rev. Microbiol. 69, 405-423 (2015).

2. Moynihan, P. J. \& Besra, G. S. Colworth prize lecture 2016: exploiting new biological targets from a whole-cell phenotypic screening campaign for TB drug discovery. Microbiology 163, 1385-1388 (2017).

3. Diacon, A. H. et al. $\beta$-Lactams against tuberculosis-new trick for an old dog? N. Engl. J. Med. 375, 393-394 (2016).

4. Deshpande, D. et al. Ceftazidime-avibactam has potent sterilizing activity against highly drug-resistant tuberculosis. Sci. Adv. 3, e1701102 (2017).

5. Cho, H., Uehara, T. \& Bernhardt, T. G. Beta-lactam antibiotics induce a lethal malfunctioning of the bacterial cell wall synthesis machinery. Cell 159, 1300-1311 (2018).

6. Santin, Y. G. \& Cascales, E. Domestication of a housekeeping transglycosylase for assembly of a Type VI secretion system. EMBO Rep. 18, 138-149 (2017).

7. Höltje, J. V. Growth of the stress-bearing and shape-maintaining murein sacculus of Escherichia coli. Microbiol. Mol. Biol. Rev. 62, 181-203 (1998)

8. Reith, J. \& Mayer, C. Peptidoglycan turnover and recycling in Gram-positive bacteria. Appl Microbiol Biotechnol. 92, 1-11 (2011).

9. Johnson, J. W., Fisher, J. F. \& Mobashery, S. Bacterial cell-wall recycling. Ann. N. Y. Acad. Sci. 1277, 54-75 (2013).

10. Cookson, B. T., Tyler, A. N. \& Goldman, W. E. Primary structure of the peptidoglycan-derived tracheal cytotoxin of Bordetella pertussis. Biochemistry 28, 1744-1749 (1989).

11. Melly, M. \& McGee, Z. Ability of monomeric peptidoglycan fragments from Neisseria gonorrhoeae to damage human fallopian-tube mucosa. J. Infect. Dis. 149, 378-386 (1984).

12. Koropatnick, T. A. et al. Microbial factor-mediated development in a hostbacterial mutualism. Science 306, 1186-1188 (2004).

13. Girardin, S. E. et al. Nod1 detects a unique muropeptide from Gram-negative bacterial peptidoglycan. Science 300, 1584-1587 (2003).

14. Goodell, E. W. Recycling of murein by Escherichia coli. J. Bacteriol. 163, 305-310 (1985).

15. Gisin, J., Schneider, A., Nägele, B., Borisova, M. \& Mayer, C. A cell wall recycling shortcut that bypasses peptidoglycan de novo biosynthesis. Nat. Chem. Biol. 9, 491-493 (2013).

16. Machowski, E. E., Senzani, S. \& Ealand, C. Comparative genomics for mycobacterial peptidoglycan remodelling enzymes reveals extensive genetic multiplicity. BMC Microbiol. 14, 75 (2014).

17. Nikitushkin, V. D. et al. A product of RpfB and RipA joint enzymatic action promotes the resuscitation of dormant mycobacteria. FEBS J. 282, 2500-2511 (2015).

18. Bellinzoni, M. et al. Structural studies suggest a peptidoglycan hydrolase function for the Mycobacterium tuberculosis Tat-secreted protein Rv2525c. J. Struct. Biol. 188, 156-164 (2014).

19. Hancock, I. C., Carman, S., Besra, G. S., Brennan, P. J. \& Waite, E. Ligation of arabinogalactan to peptidoglycan in the cell wall of Mycobacterium smegmatis requires concomitant synthesis of the two wall polymers. Microbiol. 148, 3059-3067 (2002).

20. Takeya, K., Hitsatsune, K. \& Nakashima, R. A cell-wall mucopeptide complex obtained from the culture filtrate of tubercle bacilli. Biochim. Biophys. Acta 54, 595-597 (1961).

21. Ruscitto, A. et al. Identification of a novel N-acetylmuramic acid transporter in Tannerella forsythia. J. Bacteriol. 198, 3119-3125 (2016).

22. Marrero, J., Trujillo, C., Rhee, K. Y. \& Ehrt, S. Glucose phosphorylation is required for Mycobacterium tuberculosis persistence in mice. PLoS Pathog. 9, e1003116-e1003116 (2013).

23. Cheng, Q., Li, H., Merdek, K. \& Park, J. T. Molecular characterization of the beta- $\mathrm{N}$-acetylglucosaminidase of Escherichia coli and its role in cell wall recycling. J. Bacteriol. 182, 4836-4840 (2000).

24. Lombard, V., Golaconda Ramulu, H., Drula, E., Coutinho, P. M. \& Henrissat, B. The carbohydrate-active enzymes database (CAZy) in 2013. Nucleic Acids Res. 42, D490-D495 (2013).

25. Macdonald, S. S., Blaukopf, M. \& Withers, S. G. N-Acetylglucosaminidases from CAZy family GH3 are really glycoside phosphorylases, thereby explaining their use of histidine as an acid/base catalyst in place of glutamic acid. J. Biol. Chem. 290, 4887-4895 (2015).

26. Bacik, J.-P., Whitworth, G. E., Stubbs, K. A., Vocadlo, D. J. \& Mark, B. L. Active site plasticity within the glycoside hydrolase NagZ underlies a dynamic mechanism of substrate distortion. Chem. Biol. 19, 1471-1482 (2012).
27. Vadlamani, G. et al. Conformational flexibility of the glycosidase NagZ allows it to bind structurally diverse inhibitors to suppress $\beta$-lactam antibiotic resistance. Protein Sci. 26, 1161-1170 (2017).

28. Målen, H., Pathak, S., Søfteland, T., de Souza, G. A. \& Wiker, H. G. Definition of novel cell envelope associated proteins in Triton X-114 extracts of Mycobacterium tuberculosis H37Rv. BMC Microbiol. 10, 132 (2010).

29. González-Zamorano, M. et al. Mycobacterium tuberculosis glycoproteomics based on ConA-lectin affinity capture of mannosylated proteins. J. Proteome Res. 8, 721-733 (2009).

30. Ducatti, D. R. B., Carroll, M. A. \& Jakeman, D. L. On the phosphorylase activity of GH3 enzymes: A $\beta$-N-acetylglucosaminidase from Herbaspirillum seropedicae SmR1 and a glucosidase from Saccharopolyspora erythraea. Carbohydr. Res. 435, 106-112 (2016).

31. Morimoto, K., Karita, S., Kimura, T., Sakka, K. \& Ohmiya, K. Cloning, sequencing, and expression of the gene encoding Clostridium paraputrificum chitinase $\mathrm{ChiB}$ and analysis of the functions of novel cadherin-like domains and a chitin-binding domain. J. Bacteriol. 179, 7306 (1997).

32. Acebrón, I. et al. Catalytic cycle of the N-acetylglucosaminidase NagZ from pseudomonas aeruginosa. J. Am. Chem. Soc. 139, 6795-6798 (2017).

33. Tsukamura, M. Identification of mycobacteria. Tubercle 48, 311-338 (1967).

34. Bowles, J. A. \& Segal, W. Kinetics of utilization of organic compounds in the growth of Mycobacterium tuberculosis. J. Bacteriol. 90, 157-163 (1965).

35. Khatri, B. et al. High throughput phenotypic analysis of mycobacterium tuberculosis and mycobacterium bovis strains' metabolism using biolog phenotype microarrays. PLoS ONE 8, e52673 (2013).

36. Billig, S. et al. Lactate oxidation facilitates growth of Mycobacterium tuberculosis in human macrophages. Sci. Rep. 7, e10 (2017).

37. Bardarov, S. et al. Specialized transduction: an efficient method for generating marked and unmarked targeted gene disruptions in Mycobacterium tuberculosis, M. bovis BCG and M. smegmatis. Microbiology 148, 3007-3017 (2002).

38. Zamorano, L. et al. NagZ inactivation prevents and reverts $\beta$-lactam resistance, driven by AmpD and PBP 4 mutations, in Pseudomonas aeruginosa. Antimicrob. Agents Chemother. 54, 3557-3563 (2010).

39. Laubacher, M. E. \& Ades, S. E. The Rcs phosphorelay is a cell envelope stress response activated by peptidoglycan stress and contributes to intrinsic antibiotic resistance. J. Bacteriol. 190, 2065-2074 (2008).

40. Raymond, J. B., Mahapatra, S., Crick, D. C., Martin, S. \& Pavelka, J. Identification of the nam $H$ Gene, encoding the hydroxylase responsible for the $\mathrm{N}$-glycolylation of the mycobacterial peptidoglycan. J. Biol. Chem. 280, 326-333 (2005)

41. Du, P., Sohaskey, C. D. \& Shi, L. Transcriptional and physiological changes during mycobacterium tuberculosis reactivation from non-replicating persistence. Front. Microbiol. 7, R106 (2016).

42. Ahangar, M. S. et al. Structural and functional determination of homologs of the Mycobacterium tuberculosis $\mathrm{N}$-acetylglucosamine-6-phosphate deacetylase (NagA). J. Biol. Chem. 293, 9770-9783 (2018).

43. White, G. F., Russell, N. J. \& Tidswell, E. C. Bacterial scission of ether bonds Microbiol. Rev. 60, 216-232 (1996).

44. Sauton, B. Sur la nutrition minerale du bacille tuberculeux. C. R. Acad. Sci. 155, 860-862 (1912).

45. Winter, G. xia2: an expert system for macromolecular crystallography data reduction. J. Appl. Crystallogr. 43, 186-190 (2010).

46. Winn, M. D. et al. Overview of the CCP4 suite and current developments. Acta Crystallogr. D. Biol. Crystallogr. 67, 235-242 (2011).

47. Emsley, P., Lohkamp, B., Scott, W. G. \& Cowtan, K. Features and development of Coot. Acta Crystallogr. D. Biol. Crystallogr. 66, 486-501 (2010).

48. Adams, P. D. et al. PHENIX: a comprehensive Python-based system for macromolecular structure solution. Acta Crystallogr. D Biol. Crystallogr. 66, 213-221 (2010).

49. Joosten, R. P., Long, F., Murshudov, G. N. \& Perrakis, A. The PDB_REDO server for macromolecular structure model optimization. IUCrJ 1, 213-220 (2014).

50. Maeda, H. A new lysozyme assay based on fluorescence polarization or fluorescence intensity utilizing a fluorescent peptidoglycan substrate. J. Biochem. 88, 1185-1191 (1980).

51. Moynihan, P. J. \& Clarke, A. J. Substrate specificity and kinetic characterization of peptidoglycan O-acetyltransferase B from Neisseria gonorrhoeae. J. Biol. Chem. 289, 16748-16760 (2014).

52. Jarle Horn, S. \& Eijsink, V. G. H. A reliable reducing end assay for chitooligosaccharides. Carbohydr. Polym. 56, 35-39 (2004).

53. Stover, C. K. et al. New use of BCG for recombinant vaccines. Nature 351, 456-460 (1991).

54. Shenderov, K. et al. Cord factor and peptidoglycan recapitulate the Th17promoting adjuvant activity of mycobacteria through mincle/ CARD9 signaling and the inflammasome. J. Immunol. 190, 5722-5730 (2013).

55. Chimichi, S., Boccalini, M., Cravotto, G. \& Rosati, O. A new convenient route to enantiopure 2-coumarinyloxypropanals: application to the synthesis of optically active geiparvarin analogues. Tetrahedron Lett. 47, 2405-2408 (2006). 
56. Chimichi, S. et al. Synthesis and biological evaluation of new geiparvarin derivatives. ChemMedChem 4, 769-779 (2009).

57. Kabsch, W. A solution for the best rotation to relate two sets of vectors. Acta Cryst. 32, 922-923 (1976).

\section{Acknowledgements}

We thank Sudagar S. Gurcha, Katherine Abrahams and Albel Singh for technical assistance. P.J.M. acknowledges support in the form of a Future Leader Fellowship and a David Phillips Fellowship from the UK Biotechnology and Biological Sciences Research Council (BB/N011945/1 and BB/S010122/1). G.V.M. acknowledges funding from UK Biotechnology and Biological Sciences Research Council (BB/P001513/1). A.L.L. acknowledges funding from UK Biotechnology and Biological Sciences Research Council (BB/J015229/1). G.S.B acknowledges support in the form of a Personal Research Chair from Mr. James Bardrick and the UK Medical Research Council (grant MR/S000542/1).

\section{Author contributions}

Conceived of the study: P.J.M. conceived and designed the experiments: P.J.M., G.V.M., A.L.L., G.S.B. performed the experiments: P.J.M., I.T.C, N.V., M.J., M.C. analysed the data: P.J.M., I.T.C, N.V., M.C. G.V.M., A.L.L., G.S.B. wrote the manuscript: P.J.M. edited the manuscript: P.J.M, G.V.M., A.L.L., G.S.B.

\section{Additional information}

Supplementary Information accompanies this paper at https://doi.org/10.1038/s41467019-10586-2.
Competing interests: The authors declare no competing interests.

Reprints and permission information is available online at http://npg.nature.com/ reprintsandpermissions/

Peer review information: Nature Communications thanks the anonymous reviewer(s) for their contribution to the peer review of this work.

Publisher's note: Springer Nature remains neutral with regard to jurisdictional claims in published maps and institutional affiliations.

(c) (i) Open Access This article is licensed under a Creative Commons Attribution 4.0 International License, which permits use, sharing, adaptation, distribution and reproduction in any medium or format, as long as you give appropriate credit to the original author(s) and the source, provide a link to the Creative Commons license, and indicate if changes were made. The images or other third party material in this article are included in the article's Creative Commons license, unless indicated otherwise in a credit line to the material. If material is not included in the article's Creative Commons license and your intended use is not permitted by statutory regulation or exceeds the permitted use, you will need to obtain permission directly from the copyright holder. To view a copy of this license, visit http://creativecommons.org/ licenses/by/4.0/.

(C) The Author(s) 2019 